\title{
Structure of Microemulsion-ABA Triblock Copolymer Networks
}

\author{
J. M. G. Sarraguça* and A. A. C. C. Pais \\ Department of Chemistry, University of Coimbra, 3004-353 Coimbra, Portugal \\ Per Linse \\ Physical Chemistry 1, Center for Chemistry and Chemical Engineering, Lund University,
P.O. Box 124, S-22100 Lund, Sweden
}

Received May 29, 2008. Revised Manuscript Received July 15, 2008

\begin{abstract}
Structural equilibrium properties of transient networks formed by microemulsion droplets and ABA triblock copolymers in solution have been studied by Monte Carlo simulation. The droplets were represented by soft spheres, and the polymers were represented by junctions connected by harmonic bonds with an angular potential regulating the intrinsic chain stiffness. The interaction parameters were selected such that the end A-blocks were localized inside the droplets and the middle B-block in the continuous phase. The influence of (i) the polymer concentration, (ii) the polymer stiffness, and (iii) the contour length of the middle B-block on the formation and the structure of the microemulsion-polymer network were investigated using polymer end-to-end separation probability distribution functions, droplet radial distribution functions, droplet-droplet nearest-neighbor probability distribution functions, and network connectivity indicators. An increase of the polymer-droplet number ratio had a strong impact on the network formation. Under typical conditions and at an intermediate polymer-droplet number ratio, (i) the fraction of polymers forming bridges between droplets increased from essentially zero to unity and (ii) the fraction of polymers that were forming loops decreased as the ratio of the polymer end-to-end separation and the surface-to-surface separation between neighboring droplets for a hypothetical homogeneous droplet distribution was increased from 0.5 to 2 . For long and flexible polymers, a mesoscopic segregation triggered by a depletion attraction between droplets appeared, and, furthermore, for sufficiently stiff chains, only bridge conformations occurred. The percolation probability could be represented as a function of the average droplet cluster size only, across all systems.
\end{abstract}

\section{Introduction}

It has been known for some time that the addition of hydrophobically end-capped hydrophilic polymers to an oil-inwater microemulsion changes many properties of the system significantly. Such changes have been attributed to the formation of a polymer network with the droplets as physical crosslinkers. ${ }^{1-5}$ The favorable interaction between the hydrophobic end-blocks of the polymer and the oil droplets results in most end-blocks residing in the droplets. The polymers attain essentially two types of conformations: loops where both end-blocks reside in the same droplet, and bridges where the end-blocks reside in different droplets. As bridging becomes extensive, a transient network of droplets and polymers is formed. When sufficiently extended, the network creates new viscoelastic properties, which renders these systems important in many technological applications, such as paints, cosmetics, oil recovery, and drug delivery. Furthermore, microemulsions that contain end-capped polymers provide important model systems for the study of network formation, because such systems allow control of parameters such as the number of polymer ends per node, the distance between nodes, and the length of the unperturbed linker.

**Author to whom correspondence should be addressed. Current contact information: Departamento de Química, Rua Larga, 3004-535 Coimbra, Coimbra, Portugal. Phone: +351 854466. Fax: +351 239827703. E-mail: jsarraguca@qui.uc.pt.

(1) Eicke, H.; Quellet, C.; Xu, G. Colloids Surf. 1989, 36, 97-107.

(2) Quellet, C.; Eicke, H.; Xu, G.; Hauger, Y. Macromolecules 1990, 23 3347-3352.

(3) Struis, R. P. W. J.; Eicke, H. J. Chem. Phys. 1991, 95, 5989-5996.

(4) Zölzer, U.; Eicke, H. J. Phys. II 1992, 2, 2207-2219.

(5) Vollmer, D.; Vollmer, J.; Stühn, B.; Wehrli, E.; Eicke, H. Phys. Rev. E: Stat. Phys. 1995, 52, 5146-5155.
Over the past decade, a large amount of work has been performed, with a focus on the specific characteristics of these systems, using techniques such as scattering experiments, ${ }^{6-12}$ rheology measurements, ${ }^{2,7,8,13-16}$ nuclear magnetic resonance (NMR), ${ }^{17}$ electric conductivity, ${ }^{6,13,16,18}$ electron microscopy, ${ }^{14}$ and luminescence quenching. ${ }^{16}$ Also, studies of related systems by simulation and theoretical methods have been made, focusing on structural ${ }^{19-28}$ and dynamic properties. ${ }^{29-33}$

(6) Meier, W.; Falk, A.; Odenwald, M.; Stieber, F. Colloid Polym. Sci. 1996, $274,218-226$.

(7) Bagger-Jörgenssen, H.; Coppola, L.; Thuresson, K.; Olsson, U.; Mortensen, K. Langmuir 1997, 13, 4204-4218.

(8) Michel, E.; Filali, M.; Aznar, R.; Porte, G.; Appell, J. Langmuir 2000, 16, 8702-8711.

(9) Filali, M.; Aznar, R.; Svensson, M.; Porte, G.; Appell, J. J. Phys. Chem. B 1999, 103, 7293-7301.

(10) Schwab, M.; Stühn, B. J. Chem. Phys. 2002, 112, 6461-6471.

(11) Michel, E.; Porte, G.-.; Cipelletti, L.; Appell, J. Langmuir 2004, 20, 984 990.

(12) Jansson, J.; Schillen, K.; Nilsson, M.; Söderman, O.; Fritz, G.; Bergmann, A.; Glatter, O. J. Phys. Chem. B 2005, 109, 7073-7083.

(13) Meier, W.; Eicke, H.; Odenwald, M. Colloids Surf. A 1996, 110, 287292.

(14) Meier, W.; Hotz, J.; Günther-Ausborn, S. Langmuir 1996, 12, 50285032 .

(15) Watanabe, H.; Sato, T.; Osaki, K.; Yao, M.; Yamagishi, A. Macromolecules 1997, 30, 5877-5892.

(16) Mays, H.; Almgren, M. J. Chem. Phys. B 1999, 103, 9432.

(17) Fleischer, G.; Stieber, F.; Hofmeier, U.; Eike, H. Langmuir 1994, 10, 1780-1785.

(18) Mays, H.; Almgren, M.; Brown, W. Ber. Bunsen. Phys. Chem. 1998, 102, 1648-1653.

(19) Santore, M. M.; Russel, W. B.; Prud'homme, R. K. Macromolecules 1990, 23, 3821-3832.

(20) Grest, G. S.; Kremer, K. Macromolecules 1990, 23, 4994-5000.

(21) Milner, S. T.; Witten, T. A. Macromolecules 1992, 25, 5495-5503.

(22) Groot, R. D.; Agterof, W. G. J. Chem. Phys. 1994, 100, 1649-1656.

(23) Turner, M. S. Macromolecules 1995, 28, 6878-6882.

(24) Björling, M.; Stilbs, P. Macromolecules 1998, 31, 9033-9043. 
When the polymer-droplet number ratio is increased, a fluid sol is replaced by a viscoelastic transient gel, in which the droplets act as cross-linkers for the polymer ends and the network expands over the entire sample. ${ }^{34}$ The location of the fluid-gel transition is coupled to the percolation threshold, which is primarily dependent on the connectivity of the network and less on the adhesion free energy of the hydrophobic end-blocks. ${ }^{34}$ According to the geometrical theory of percolation (see ref 35 and references therein), the number of bridges per droplet at the percolation threshold is 1.5. However, different values for this threshold concentration have experimentally been obtained, ranging from 2 to $4 .^{2,8,36}$ Sometimes, a distinction between two different types of percolation, related to geometrical connectivity and structural rigidity, respectively, have been made. ${ }^{8}$

The residence time of the end-blocks of a chain in a droplet is controlled by the adsorption energy. ${ }^{8}$ Although the end-blocks are essentially confined to the droplets, an end-block still possesses some finite probability to change droplet. Such a change of droplet affiliation has been proposed to occur essentially by two different mechanisms: (i) a passage of the end-block from one droplet to the other during a collision of the two droplets and (ii) a passage through the continuous medium, ${ }^{37}$ possibly stabilized by small amounts of surfactant. ${ }^{38}$ In the former case, the residence time of an end-block in an droplet is controlled by the droplet collision rate. $^{17}$

The influence of the middle and end-blocks length of the block copolymer on the characteristics of the systems has been extensively studied..$^{34,36,39,40}$ The length of the end-block has been shown to affect the properties of the systems significantly. For example, an increase of a hydrophobic end-blocks from 12 $\mathrm{CH}_{2}$ groups to $18 \mathrm{CH}_{2}$ groups increases the time scale at which the rupture of the bridging occurs by 3 orders of magnitude in an oil-in-water microemulsion. ${ }^{34,36}$ The length of the middle block is important for the network structure, because it determines the distance between directly connected droplets. ${ }^{35}$

In this work, we investigate solutions that contain microemulsion droplets and ABA triblock copolymers using Monte Carlo computer simulations. Our focus is on the influence of (i) polymer concentration, (ii) intrinsic stiffness of the polymers, and (iii) midblock length on the structure of the solution. Parameters have been selected to represent end-capped hydrophilic polymers in an oil-in-water emulsion. However, our generic results are, of course, equally valid for hydrophillically endcapped hydrophobic polymers in a water-in-oil microemulsion.

(25) Karastasos, K.; Anastasiadis, S. H.; Pakula, T.; Watanabe, H. Macromolecules 2000, 33, 523-541.

(26) Bhatia, S. R.; Russel, W. B. Macromolecules 2000, 33, 5713-5720.

(27) Zilman, A.; Kieffer, J.; Molino, F.; Porte, G.; Safran, S. A. Phys. Rev. Lett. 2003, 91, 015901-14.

(28) Hed, G.; Safran, S. A. Eur. Phys. J. E 2006, 19, 69-76.

(29) Groot, R. D.; Agterof, W. G. M. J. Chem. Phys. 1994, 100, 1657-1664

(30) Groot, R. D.; Agterof, W. G. M. Macromolecules 1995, 28, 6284-6295.

(31) Huh, J.; Balazs, A. C. J. Chem. Phys. 2000, 113, 2025-2031.

(32) Pellens, L.; Ahn, K. H.; Lee, S. J.; Mewis, J. J. Non-Newtonian Fluid Mech. 2004, 121, 87-100.

(33) Surve, M.; Pryamitsyn, V.; Ganesan, V. J. Chem. Phys. 2006, 125, 064903.

(34) Filali, M.; Ouazzani, M. J.; Michel, E.; Aznar, R.; Porte, G.; Appell, J.

J. Phys. Chem. B 2001, 105, 10528-10535.

(35) Molino, F.; Appell, J.; Filali, M.; Michel, E.; Porte, G.; Mora, S.; Sunyer, E. J. Phys.: Condens. Matter 2000, 12, 491-498.

(36) Michel, E.; Cipelletti, L.; d'Humieres, E.; Gambin, Y.; Urbach, W.; Porte, G.; Appell, J. Phys. Rev. E: Stat. Phys. 2002, 66, 031402-17. 5074.

(37) Odenwald, M.; Eicke, H.; Meier, W. Macromolecules 1995, 28, 5069-

(38) Stieber, F.; Hofmeier, U.; Eicke, H.; Fleisher, J. Ber. Bunsen. Phys. Chem. 1993, 97, 812-818.

(39) Antunes, F. E.; Thuresson, K.; Lindman, B.; Miguel, M. G. Colloids Surf. A 2003, 215, 87-100.

(40) Lynch, I.; Picullel, L. J. Phys. Chem. B 2004, 108, 7515-7522.

(41) Linse, P. Molsim, Version 4.0.3, Lund University: Lund, Sweden, 2004.

(42) Akinchina, A.; Linse, P. Macromolecules 2002, 35, 5183-5193.
The results highlight various important aspects in the formation of networks in this type of system. For chains with intermediate stiffness, the amount of polymer has a strong influence on the overall connectivity, despite an essentially constant fraction of loops and bridges. The fraction of polymers that form bridges was determined to have a strong dependence on the length ratio of the polymer root-mean-square (rms) end-to-end separation and the surface-to-surface separation between neighboring droplets for a lattice arrangement of the droplets. Multiple bridging between droplets is visible before a single cluster involving all droplets is formed. The presence of very stiff or long chains substantially influences the droplet distribution and may induce mesoscopic segregation in the second case.

\section{Model}

A coarse-grained approach is taken to model solutions that contain oil-in-water microemulsions and hydrophobically endcapped hydrophillic polymers. The droplets are represented by soft spheres characterized by the radius $R_{\mathrm{d}}=80 \AA$. The ABAtriblock copolymer is built up of segments connected by harmonic bonds. An end A-block is represented by one hydrophobic segment, and the middle B-block by $N_{\mathrm{B}}$ hydrophilic segments. Because the excluded-volume effect of the polymer segments is unimportant in the present investigation, the polymer description is further simplified by representing each segment by a point. The stiffness of the polymers is regulated using an angular potential.

In more detail, the total potential energy of the solution is composed of five terms, as follows:

$$
U=U_{\text {seg,seg }}+U_{\text {seg,d }}+U_{\text {d,d }}+U_{\text {bond }}+U_{\text {ang }}
$$

where the three first terms represent intermolecular contributions and the last two represent intrapolymer contributions.

First, we have assumed $U_{\text {seg,seg }}=0$; thus, there is no segment-segment interaction and, in particular, no effective attraction between the end-blocks in the aqueous phase. In these systems, the solvation of the end-blocks inside the oil droplets dominates over the polymer self-association.

The total segment-droplet potential energy $U_{\text {seg,d }}$ is given by

$$
U_{\mathrm{seg}, \mathrm{d}}=\sum_{i=1}^{N_{\mathrm{p}} N_{\mathrm{seg}}} \sum_{j=1}^{N_{\mathrm{d}}} \epsilon_{\mathrm{seg}, \mathrm{d}}\left[\frac{1}{2} \pm \frac{\arctan \left(r_{i j}-R_{\mathrm{d}}\right)}{\pi}\right]
$$

where $N_{\mathrm{p}}$ denotes the number of polymers, $N_{\text {seg }}$ the number of segments in one polymer $\left(N_{\text {seg }}=2+N_{\mathrm{B}}\right), N_{\mathrm{d}}$ the number of droplets, and $r_{i j}$ the center-to-center separation between segment $i$ and droplet $j$. Furthermore, in eq 2, the minus sign applies to A-segments and the plus sign to B-segments. Throughout, we have used a value of $\epsilon_{\mathrm{seg}, \mathrm{d}}=30 \mathrm{~kJ} / \mathrm{mol}$. Thus, we assume the same potential energy cost (i) to transfer a hydrophobic A-segment from the oil droplet to the continuous aqueous phase and (ii) to transfer a hydrophillic B-segment from the aqueous phase to a droplet. With the experimental value of $1.2 k_{\mathrm{B}} T$ for transferring a $\mathrm{CH}_{2}$ group from oil to water, ${ }^{34,36}$ our value of $\epsilon_{\mathrm{seg}, \mathrm{d}}$ implies that one A-segment energetically represents a block of ca. $10 \mathrm{CH}_{2}$ groups.

The droplets interact through a soft repulsive potential, for simplicity, employing the same functional form as that used for the B-segment-droplet interaction. The total droplet-droplet potential energy $U_{\mathrm{d}, \mathrm{d}}$ is given by

$$
U_{\mathrm{d}, \mathrm{d}}=\sum_{i=1}^{N_{\mathrm{d}}} \sum_{j=i+1}^{N_{\mathrm{d}}} \epsilon_{\mathrm{d}, \mathrm{d}}\left[\frac{1}{2}-\frac{\arctan \left(r_{i j}-2 R_{\mathrm{d}}\right)}{\pi}\right]
$$

where $r_{i j}$ denotes the center-to-center separation between droplet $i$ and droplet jand $\epsilon_{\mathrm{d}, \mathrm{d}}=100 \mathrm{~kJ} / \mathrm{mol}$. Experimentally, micro- 
Table 1. Parameters and Key Properties of the Systems Investigated ${ }^{a}$

\begin{tabular}{|c|c|c|c|c|c|c|c|}
\hline system & $\beta$ & $k_{\text {ang }}\left(\mathrm{J} / \mathrm{K}^{2}\right)$ & $N_{\mathrm{B}}$ & $\left\langle R_{\mathrm{ee}}\right\rangle^{1 / 2}(\AA)^{b}$ & $\left\langle R_{\mathrm{g}}{ }^{2}\right\rangle^{1 / 2}(\AA)^{b}$ & $l_{\mathrm{p}}(\AA)^{b, c}$ & $\left\langle R_{\mathrm{ee}}^{2}\right\rangle^{1 / 2} / R_{\mathrm{d}, \mathrm{d}}^{\text {surf }}$ \\
\hline reference & 3 & $3.4 \times 10^{-24}$ & 20 & $155.0(0.3)$ & $50.4(0.1)$ & 51.1 & 1.29 \\
\hline Ia & 1 & & & & & & \\
\hline $\mathrm{Ib}$ & 2 & & & & & & \\
\hline Ic & 4 & & & & & & \\
\hline Id & 6 & & & & & & \\
\hline IIa & & 0 & & $69.7(0.2)$ & $29.1(0.1)$ & 15.2 & 0.58 \\
\hline IIb & & $1.7 \times 10^{-23}$ & & $252.9(0.2)$ & $83.0(0.1)$ & 211 & 2.11 \\
\hline IIIa & & & 10 & $104.3(0.2)$ & $38.2(0.1)$ & 51.0 & 0.87 \\
\hline IIIb & & & 30 & $192.6(0.3)$ & $74.0(0.1)$ & 51.1 & 1.63 \\
\hline IIIc & & & 40 & $224.5(0.4)$ & $87.3(0.1)$ & 51.2 & 1.87 \\
\hline IVa & & $1.7 \times 10^{-23}$ & 5 & $84.8(0.1)$ & $28.9(0.1)$ & 212 & 0.72 \\
\hline $\mathrm{IVb}$ & & $1.7 \times 10^{-23}$ & 10 & $146.8(0.1)$ & $48.2(0.1)$ & 211 & 1.22 \\
\hline
\end{tabular}

${ }^{a}$ Values are provided for systems I-IV as long as they differ from those of the reference system. ${ }^{b}$ For droplet-free polymer solutions. Because the polymers are noninteracting, these results were obtained by simulating a single polymer. ${ }^{c}$ Evaluated according to $l_{\mathrm{p}}=\left\langle R_{\mathrm{bb}}{ }^{2}\right\rangle^{1 / 2} /(1+\langle\cos \theta\rangle)$, where $\left\langle R_{\mathrm{bb}}{ }^{2}\right\rangle^{1 / 2}$ denotes the root-mean-square segment-segment separation and $\theta=180^{\circ}-\alpha$. (See ref 42 ).

emulsion solutions display a slow change of the droplet number density and droplet size through different processes. Here, we assume the number density and size to be constant.

The total bond potential energy $U_{\text {bond }}$ is given by

$$
U_{\text {bond }}=\sum_{m=1}^{N_{\text {bond }}} \frac{k_{\text {bond }}}{2}\left(r_{m, \text { bond }}-r_{0}\right)^{2}
$$

where $N_{\text {bond }}$ is the number of bonds in the system $\left(N_{\text {bond }}=N_{\mathrm{p}^{-}}\right.$ $\left.\left(N_{\text {seg }}-1\right)\right), r_{m \text {,bond }}$ the bond length of bond $m, k_{\text {bond }}$ the harmonic force constant $\left(k_{\text {bond }}=0.4 \mathrm{~N} / \mathrm{m}^{2}\right)$, and $r_{0}$ the equilibrium bond distance $\left(r_{0}=15 \AA\right)$.

Finally, the total angular potential energy $U_{\text {ang }}$ is given by

$$
U_{\text {ang }}=\sum_{m=2}^{N_{\text {ang }}} \frac{k_{\text {ang }}}{2}\left(\alpha_{m, \text { ang }}-\alpha_{0}\right)^{2}
$$

where $N_{\text {ang }}$ is the number of angles formed by three consecutive segments in a chain in the system $\left(N_{\text {ang }}=N_{\mathrm{p}}\left(N_{\text {seg }}-2\right)\right), \alpha_{m}$, ang the measure of angle $m, k_{\text {ang }}$ the angular force constant, and $\alpha_{0}$ the equilibrium angle $\left(\alpha_{0}=180^{\circ}\right)$.

An important parameter that characterizes the investigated systems is the polymer-droplet number ratio, $\beta$ :

$$
\beta=\frac{N_{\mathrm{p}}}{N_{\mathrm{d}}}
$$

Throughout, $N_{\mathrm{d}}=20$ have been used, corresponding to a droplet volume fraction $\phi_{\mathrm{d}}=0.1$. If the droplets were distributed on a primitive cubic lattice, a nearest-neighbor droplet-droplet separation of $R_{\mathrm{d}, \mathrm{d}}=\left[\left(4 \pi R_{\mathrm{d}}{ }^{3}\right) /\left(3 \phi_{\mathrm{d}}\right)\right]^{1 / 3} \approx 280 \AA$ and a corresponding surface-to-surface separation of $R_{\mathrm{d}, \mathrm{d}}^{\text {surf }}=R_{\mathrm{d}, \mathrm{d}}-2 R_{\mathrm{d}}=120 \AA$ are obtained.

In the present study, we have systematically varied (i) the number of polymers $N_{\mathrm{p}}$ and, hence, the polymer-droplet number ratio $\beta$, (ii) the chain flexibility as described by its intrinsic persistence length $l_{\mathrm{p}}$, and (iii) the contour length of the B-block using different values of $N_{\mathrm{B}}$. One of the systems will be referred to as the reference system, characterized by $\beta=3, l_{\mathrm{p}}=51.1 \AA$, and $N_{\mathrm{B}}=20$. Here, the contour length of a relaxed polymer becomes $r_{0}\left(N_{\text {seg }}-1\right)=315 \AA$. In addition, we have also considered a polymer-free droplet solution $(\beta=0)$ and dropletfree polymer solutions. Table 1 provides a summary of parameters and key properties of the systems investigated. Throughout, a temperature of $T=298 \mathrm{~K}$ has been used.

\section{Method}

The properties of the systems were obtained by performing the Metropolis Monte Carlo simulation in the canonical ensemble (with a constant number of particles, volume, and temperature). The systems investigated were confined in a box with the edge length $L=756 \AA$ and with periodic boundary conditions applied. All particles (droplets and segments) were subjected to independent translational trial moves. Furthermore, pivot rotations of a subchain of the polymer and translations of entire chains were also made. Finally, to improve the sampling efficiency further, cluster moves involving droplets and end-segments confined in that droplet were also used. Equilibration runs comprised at least $2.5 \times 10^{5}$ steps, and production runs involved $5 \times 10^{5}$ steps. One step encompasses $N$ trial moves, where $N$ $=N_{\mathrm{d}}+N_{\mathrm{p}} N_{\text {seg }}$ is the total number of particles in the system. The simulations were performed using the Molsim package. ${ }^{41}$

The spatial extensions of the polymers were analyzed through probability distribution functions of the polymer end-to-end separation $P\left(R_{\mathrm{ee}}\right)$ and the radius of gyration $P\left(R_{\mathrm{g}}\right)$, of which most attention will be payed to the former. Furthermore, a polymer was classified in one of four conformations: (i) loop characterized by both end-blocks residing in the same droplet, (ii) bridge in which the two end-blocks reside in different droplets, (iii) tail where one end-block resides in a droplet and the other in the aqueous phase, and (iv) nonadsorbed where both end-blocks are in the aqueous phase. The fraction of polymers in conformation $x$ will be denoted by $f_{x}$ (where $x=$ loop, bridge, tail, or nonads). Generally, the fraction of nonadsorbed polymers $f_{\text {nonads }}$ was negligible, and the fraction of tails was small $f_{\text {tail }}<0.03$.

The droplet structure was examined through the droplet-droplet radial distribution function $g(r)$. It is zero at short center-tocenter separations at which the droplets overlap, and it was conventionally defined to approach unity at separations at which the spatial correlation is lost. Furthermore, the appearance of short droplet-droplet separations has been characterized by the droplet-droplet nearest-neighbor separation probability distribution $P(r)$. This function provides the distribution of nearestneighbor separations among the droplets.

The connectivity of the systems has been characterized using four connectivity indicators. Two droplets were considered to belong to the same cluster if they were connected directly or indirectly by at least one bridging polymer linking pairs of droplets. A cluster was classified according to how many droplets it contained. From the frequency of clusters with different sizes, 
two normalized cluster size distributions $P_{i}^{\mathrm{n}}$ and $P_{i}^{\mathrm{m}}$ were calculated according to

$$
P_{i}^{\mathrm{n}}=\frac{\left\langle n_{i}\right\rangle}{\sum_{i}\left\langle n_{i}\right\rangle}
$$

and

$$
P_{i}^{\mathrm{m}}=\frac{i\left\langle n_{i}\right\rangle}{\sum_{i} i\left\langle n_{i}\right\rangle}
$$

where $\left\langle n_{i}\right\rangle$ is the average number of clusters that contain $i$ droplets. Note that $P_{i}^{\mathrm{n}}$ is a "number"-weighted measure of the cluster size distribution representing that any given cluster, randomly selected, will contain $i$ droplets, whereas $P_{i}^{\mathrm{m}}$ is a "mass"-weighted measure representing the probability that any droplet chosen randomly will be found in a cluster containing $i$ droplets. The first two connectivity indicators are

$$
\left\langle N_{\mathrm{d}}^{\mathrm{n}}\right\rangle=\sum_{i} i P_{i}^{\mathrm{n}}
$$

and

$$
\left\langle N_{\mathrm{d}}^{\mathrm{m}}\right\rangle=\sum_{i} i P_{i}^{\mathrm{m}}
$$

representing the average cluster size, again "number" and "mass"weighted, respectively. Furthermore, the local connectivity was characterized by two indicators. They are the average number of droplets that are linked to a droplet $\left(\left\langle N_{\mathrm{d}}^{\text {link }}\right\rangle\right)$ and the (related) average number of bridging polymers establishing the link between two linked droplets ( $\left\langle N_{\mathrm{p}}^{\mathrm{link}}\right\rangle$, also referred to as the bond order). Obviously, we have the relation $\left\langle N_{\mathrm{p}}^{\text {link }}\right\rangle=2 \beta f_{\text {bridge }} /\left\langle N_{\mathrm{d}}^{\text {link }}\right\rangle$. Moreover, the smallest value of $\left\langle N_{\mathrm{p}}^{\text {link }}\right\rangle$ is unity and, hence, the maximal value of $\left\langle N_{\mathrm{d}}^{\mathrm{ink}}\right\rangle$ is $2 \beta f_{\text {bridge }}$, which are achieved when all linked droplet pairs are connected with only one bridging polymer per link.

\section{Results}

4.1. Effect of Polymer-Droplet Number Ratio. We will first examine the solution structure for various systems with polymer-droplet number ratios in the range of $\beta=1-6$ with a polymer having $N_{\mathrm{B}}=20$ hydrophilic segments and the intermediate persistence length of $l_{\mathrm{p}}=51.1 \AA$. In addition, some properties of droplet-free and of polymer-free solutions will also be considered.

Figure 1a displays the polymer end-to-end separation probability distribution $P\left(R_{\mathrm{ee}}\right)$ for systems with $\beta=1$ (system Ia), $\beta=3$ (the reference system), and $\beta=6$ (system Id) and for a droplet-free polymer solution. As for the latter, $P\left(R_{\mathrm{ee}}\right)$ possesses a single maximum and an almost Gaussian behavior with a mostprobable end-to-end separation of $R_{\mathrm{ee}} \approx 150 \AA$ and a rms endto-end separation of $\left\langle R_{\mathrm{ee}}{ }^{2}\right\rangle^{1 / 2}=155 \AA$. In the presence of droplets, $P\left(R_{\mathrm{ee}}\right)$ changes character. Two peaks appear, with their maxima located at $R_{\mathrm{ee}} \approx 55$ and $190 \AA$, respectively. The amplitude, width, and locations of the two maxima are essentially independent of $\beta$. Furthermore, Table 2 shows that, for these systems, the fraction of loop conformations is $f_{\text {loop }} \approx 0.41$ and the fraction of bridge conformations is $f_{\text {bridge }} \approx 0.58$, which are both independent of $\beta$, whereas the fraction of tail conformations is small $\left(f_{\text {tail }} \approx 0.01\right)$. Because (i) essentially only loop and bridge conformations occur and (ii) the maximum in $P\left(R_{\mathrm{ee}}\right)$ appearing at $R_{\mathrm{ee}}=190 \AA$ is larger than twice the droplet radius $\left(2 R_{\mathrm{d}}=160\right.$ $\AA$ ), we conclude that the maximum at the larger separation originates from polymers in bridge conformations, whereas the maximum at the smaller separation occurs from polymers in

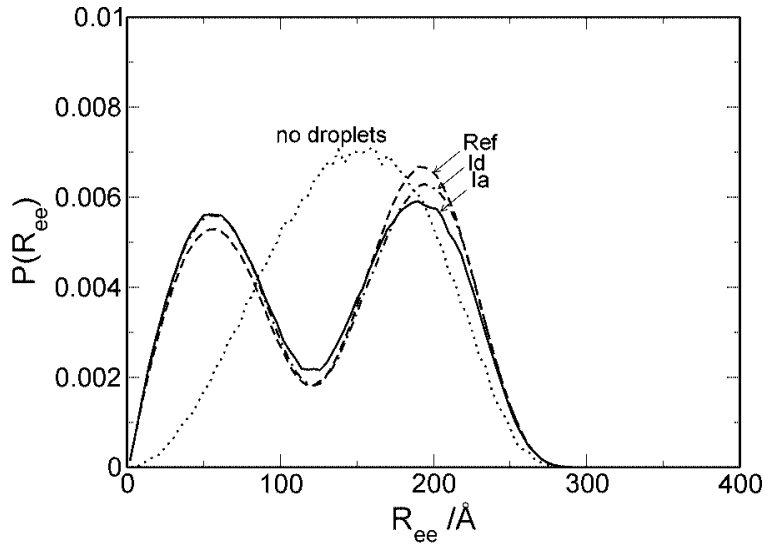

(a)

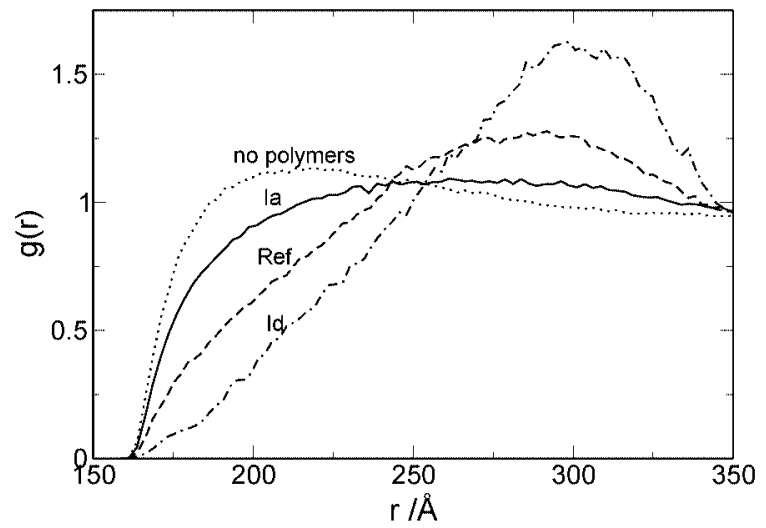

(b)

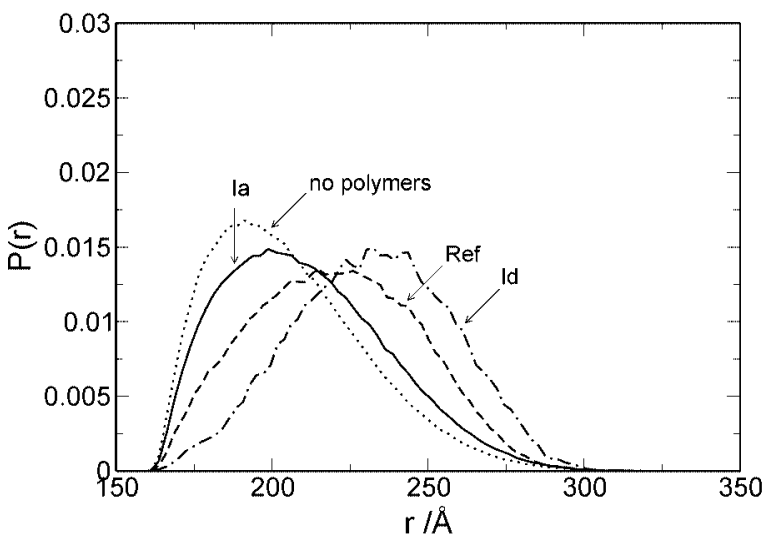

(c)

Figure 1. (a) Polymer end-to-end separation probability distribution $P\left(R_{\mathrm{ee}}\right)$, (b) droplet-droplet radial distribution function $g(r)$, and (c) droplet-droplet nearest-neighbor separation probability distribution $P(r)$ for indicated systems with different numbers of polymers.

loop conformations. Finally, from geometrical arguments, it is reasonable that a polymer with an unperturbed rms end-to-end separation of $155 \AA$ attains a typical end-to-end separation of $\sim 55 \AA$ when forming a loop on a sphere with the radius of 80 $\AA$. Thus, (i) all chains are doubly connected to droplets either in loop or bridge conformations; (ii) the spatial extensions of polymers in these two conformations are well-separated; and (iii) the chain conformations, as well as their probabilities, are essentially independent of the polymer-droplet number ratio.

The droplet-droplet radial distribution function $g(r)$ for systems with different number of polymers, including the 
Table 2. Fraction of Polymers in Loop, Bridge, and Tail Conformations, as Well as Connectivity Indicators, of the Systems Investigated

\begin{tabular}{lccccccc}
\hline system & $f_{\text {loop }}$ & $f_{\text {bridge }}$ & $f_{\text {tail }}$ & $\left\langle N_{\mathrm{d}}^{\mathrm{n}}\right\rangle$ & $\left\langle N_{\mathrm{d}}^{\mathrm{m}}\right\rangle$ & $\left\langle N_{\mathrm{d}}^{\text {link }}\right\rangle$ & $\left\langle N_{\mathrm{p}}^{\text {link }}\right\rangle$ \\
\hline reference & 0.402 & 0.585 & 0.013 & 11.9 & 18.6 & 2.80 & 1.26 \\
Ia & 0.427 & 0.559 & 0.014 & 2.02 & 4.40 & 1.03 & 1.09 \\
Ib & 0.418 & 0.562 & 0.012 & 5.63 & 13.9 & 1.91 & 1.18 \\
Ic & 0.423 & 0.561 & 0.011 & 15.9 & 19.5 & 3.38 & 1.33 \\
Id & 0.404 & 0.580 & 0.014 & 19.6 & 20.0 & 4.70 & 1.49 \\
IIa & 0.889 & 0.091 & 0.020 & 1.33 & 1.73 & 0.49 & 1.12 \\
IIb & 0.017 & 0.972 & 0.011 & 17.9 & 19.8 & 4.55 & 1.28 \\
IIIa & 0.635 & 0.356 & 0.010 & 3.84 & 9.24 & 1.57 & 1.36 \\
IIIb & 0.363 & 0.620 & 0.017 & 5.82 & 15.4 & 2.77 & 1.35 \\
IIIc & 0.473 & 0.500 & 0.027 & 2.33 & 7.96 & 1.52 & 2.00 \\
IVa & 0.003 & 0.944 & 0.013 & 18.5 & 19.4 & 2.52 & 2.35 \\
IVb & 0.004 & 0.987 & 0.009 & 19.9 & 20.0 & 3.60 & 1.65
\end{tabular}

polymer-free droplet solution $(\beta=0)$, is shown in Figure 1b. Throughout, $g(r)=0$ for $r \lesssim 160 \AA$, which is consistent with a droplet radius of $R_{\mathrm{d}}=80 \AA$. In the polymer-free droplet solution, $g(r)$ displays a weak maximum at $r \approx 210 \AA$ and then smoothly approaches unity, which is typical for a dilute solution of soft spheres that possess excluded-volume interactions only. As the number of polymers increase (increasing $\beta$ ), the maximum of $g(r)$ is shifted to larger $r$. At $\beta \geq 4$, the location of the maximum has converged to $r \approx 300 \AA$. This distance is comparable to $R_{\mathrm{d}, \mathrm{d}}$ $=280 \AA$, the nearest-neighbor droplet separation for a primitive cubic lattice distribution of the droplets. Thus, the distribution of the droplets in the solution undergoes a drastic change upon the addition of the triblock copolymers. In the absence of the polymers, droplets come into direct contact and the solution displays no long-range structure, which are conventional features of solutions of excluding volume objects at not too high volume fraction. At a polymer-droplet number ratio of $\beta=1$, significant changes have appeared, and at $\beta=6$, the solution is better described as being composed of a mesh of connected and effectively repelling droplets, which are connected by bridging polymers acting as springs.

The corresponding nearest-neighbor separation probability distributions are given in Figure 1. Without polymers, the droplets are able to approach each other up to a distance of $r=2 R_{\mathrm{d}}$ with essentially no repulsion. That leads to half of the droplets having at least a neighbor within a distance of $r=210 \AA$. As polymers are introduced and at increasing number of polymers, this distribution is shifted to longer distances, implying an effective repulsion between nearby droplets. At $\beta=6$, the average distance to the nearest neighbor is $240 \AA$. This is still smaller than the distance $R_{\mathrm{d}, \mathrm{d}}=280 \AA$, which is applicable for a primitive cubic lattice distribution, demonstrating that the network of connected droplets possesses significant flexibility.

Figure 2 displays the "mass"-weighted cluster size probability distribution. At $\beta=1$, the distribution is monotonically decaying, implying that unconnected or weakly connected droplets dominate. However, at $\beta=2$, the distribution has changed character. It is now bimodal, with a strong dominance for large clusters, implying that we have a large single network that is comprised almost entirely of droplets in equilibrium with a few clusters, many of them being unconnected droplets. At $\beta=3$, the cluster size probability distribution has a peak at $N_{\mathrm{d}}^{\mathrm{m}}=20$ and displays nonzero values only for $N_{\mathrm{d}}^{\mathrm{m}}=1$ and $N_{\mathrm{d}}^{\mathrm{m}}>15$. Thus, it seems that a transition from a solution of dispersed small clusters to a solution with extended networks appears between $\beta=1$ and $\beta=2$. Such a transition should experimentally be observed, e.g., by a strong increase of the solution viscosity.

Table 2 summarizes the results of the indicators that characterize the connectivity of the systems. As the polymer-

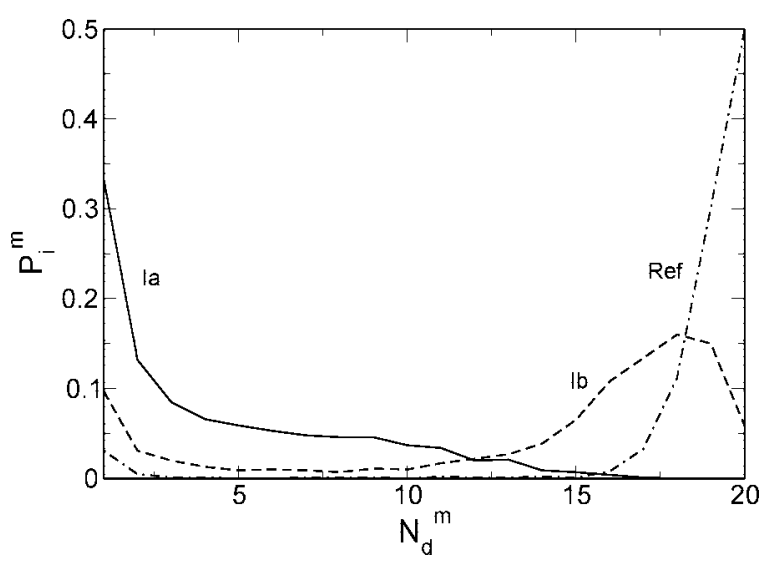

Figure 2. Cluster size probability distribution $P_{i}^{\mathrm{m}}$ for the indicated systems, containing different number of polymers.

droplet number ratio $\beta$ increases from 1 to 6 , both $\left\langle N_{\mathrm{d}}^{\mathrm{n}}\right\rangle$ and $\left\langle N_{\mathrm{d}}^{\mathrm{m}}\right\rangle$ show that the probability of clusters with larger sizes is promoted. For example, the "mass"-weighted cluster size increases from $\left\langle N_{\mathrm{d}}^{\mathrm{m}}\right\rangle=4.40$ droplets at $\beta=1$ to 13.9 droplets at $\beta=2$ and 18.6 droplets at $\beta=3$; hence, at $\beta \geq 3$, all droplets basically are connected into a single cluster. Now turning to the local connectivity indicators, $\left\langle N_{\mathrm{d}}^{\text {ink }}\right\rangle$ shows that, at $\beta=1$, each droplet is, on average, linked to one other droplet. At increasing $\beta,\left\langle N_{\mathrm{d}}^{\text {link }}\right\rangle$ increases, but slower than $\beta$ (at an approximately constant value of $f_{\text {bridge }}$ ), showing that (i) a droplet is linked to an increasing number of other droplets and (ii) an increasing frequency of links is made of more than one bridging polymer. Finally, $\left\langle N_{\mathrm{p}}^{\text {link }}\right\rangle$ shows that, at $\beta=6$, each link is, on average, made of 1.5 bridging polymers. At a sufficiently large value of $\beta$, we expect $\left\langle N_{\mathrm{d}}^{\text {link }}\right\rangle$ to become independent of $\beta$, which should appear when a droplet is fully linked to other droplets located within the distance of one end-to-end separation of a polymer. With $\left\langle R_{\mathrm{ee}}{ }^{2}\right\rangle^{1 / 2} / R_{\mathrm{d}, \mathrm{d}}^{\mathrm{surf}}$ $=1.3$, a droplet can basically only be linked to other droplets located in its first coordination shell. An addition of polymers when $\left\langle N_{\mathrm{d}}^{\text {link }}\right\rangle$ has leveled off leads only to increased multiple bridging between droplets.

4.2. Effect of Chain Flexibility. The influence of the chain flexibility on the solution properties will now be examined. In addition to the reference system, in which polymers possessed an intermediate persistence length of $l_{\mathrm{p}}=51.1 \AA$ (here, referred to as semiflexible chains), we will consider flexible chains with $l_{\mathrm{p}}=15.2 \AA$ (system IIa) and stiff chains with $l_{\mathrm{p}}=211 \AA$ (system IIb), all under the conditions of $\beta=3$ and $N_{\mathrm{B}}=20$.

Figure $3 \mathrm{a}$ displays the end-to-end separation probability distribution $P\left(R_{\mathrm{ee}}\right)$ for the systems with different chain flexibility, as well as for the corresponding droplet-free polymer solutions. In the absence of droplets, for the flexible chains, the most probable end-to-end separation becomes $65 \AA$ and $\left\langle R_{\mathrm{ee}}{ }^{2}\right\rangle^{1 / 2}=69.7 \AA$, which is consistent with $\left(N_{\text {seg }}-1\right)^{1 / 2} r_{0}=68 \AA$. Furthermore, for the stiff chains, the most-probable end-to-end separation becomes $280 \AA$, which is close to but still shorter than the contour length, $\left(N_{\text {seg }}-1\right) r_{0}=315 \AA$. In the presence of droplets, for the flexible chains, $P\left(R_{\mathrm{ee}}\right)$ displays a prominent maximum at $R_{\mathrm{ee}}$ $=25 \AA$ and a weak maximum at $R_{\mathrm{ee}}=110 \AA$. Table 2 also shows that the fraction of loop conformations is $f_{\text {loop }}=0.89$ and that of bridge conformations $i s f_{\text {bridge }}=0.09$. Regarding the stiff chains, $P\left(R_{\mathrm{ee}}\right)$ displays one prominent maximum at $R_{\mathrm{ee}} \approx 285$ $\AA$ and a very weak one at $R_{\mathrm{ee}} \approx 100 \AA$. Here, the fraction of bridge and loop conformations are 0.97 and 0.02 , respectively. Obviously, there are again gratifying correspondences between the areas of the two peaks and the fraction of chains in loop and bridge conformations. Thus, with flexible polymers, we have 


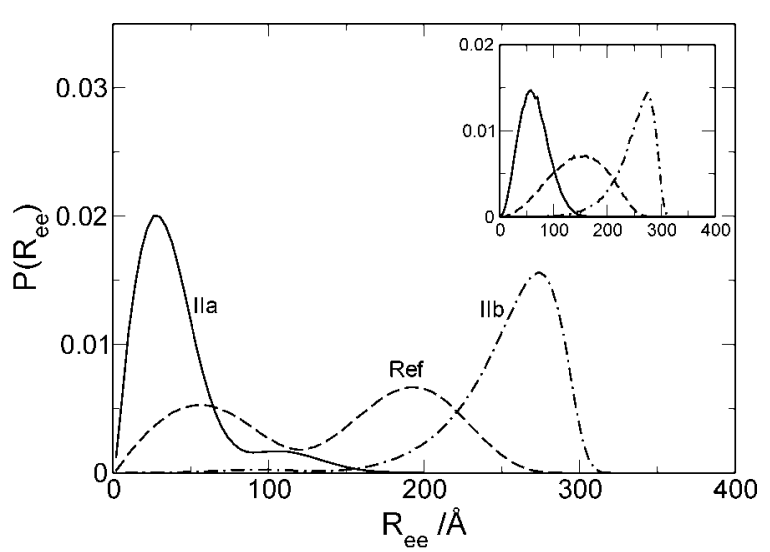

(a)

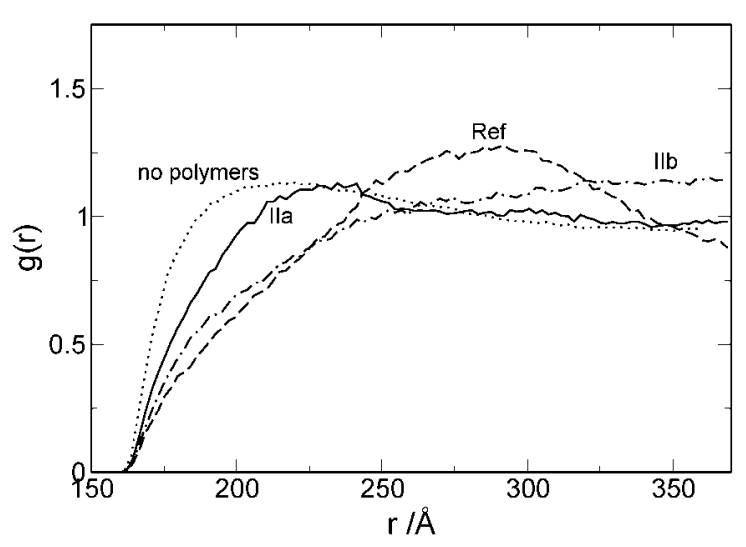

(b)

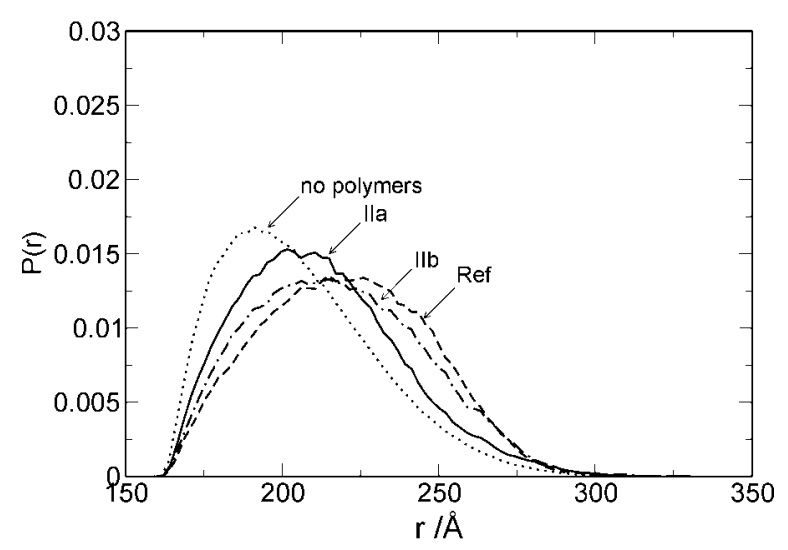

(c)

Figure 3. (a) Polymer end-to-end separation probability distribution $P\left(R_{\mathrm{ee}}\right)$, (b) droplet-droplet radial distribution function $g(r)$, and (c) droplet-droplet nearest-neighbor separation probability distribution $P(r)$ for the indicated systems containing polymers with different stiffness. In panel a, the inset shows the corresponding data for a droplet-free polymer solution.

predominantly loop conformations and with stiff polymers, we obtain bridge conformations, in both cases with the polymers adopting an end-to-end separation distribution similar to that for an unperturbed polymer, whereas with the semiflexible polymers, an almost-equal amount of loop and bridge conformations occurs.

The corresponding droplet-droplet radial distribution functions are shown in Figure 3b. As compared to a polymer-free droplet solution, when flexible chains are present, the density of droplets near a given droplet is reduced, whereas the solution structure beyond the first maximum of $g(r)$ is unaffected. The suppression of the probability of nearby droplets is attributed to the appearance of polymers partially covering the droplet surfaces, giving rise to a steric repulsion. On the other hand, with stiff polymers, (i) $g(r)$ is suppressed at short separation, more than with flexible polymers but less than with semiflexible chains, and (ii) the maximum of $g(r)$ is flat and appears at a large separation $(r \approx$ $350 \AA$ ), which is larger than where the maximum appears for semiflexbile chains. An explanation will be given below. Thus, it is clear that the chain flexibility also has implications on the droplet structure in the solution.

Figure 3 shows the nearest-neighbor probability distributions for the same set of systems. With flexible chains, the maximum is slightly shifted to longer separation, compared to a polymerfree droplet solution, supporting the notion that polymers that are anchored in loop conformations cause a short-range repelling force between droplets. This trend of displaying the maximum of $P(r)$ to longer separation continues with semiflexible chains, but is reversed with the stiff chains, which is consistent with the behavior of $g(r)$ at short separations. Our conclusion is that stiff chains with an end-to-end separation of $r \approx 350 \AA$ no longer predominantly bridge nearest-neighboring droplets; instead, the polymers bridge droplets, which are structurally further apart. The smaller frequency of neighboring droplets being bridged makes it possible for them to again be near each other.

The connectivity indicators in Table 2 show a strong dependence on the chain stiffness. In the presence of the flexible polymers, the average cluster size is $\left\langle N_{\mathrm{d}}^{\mathrm{m}}\right\rangle=1.73$, which is smaller than that for semiflexible polymers at a 3 -fold smaller polymer concentration. Furthermore, $\left\langle N_{\mathrm{d}}^{\text {link }}\right\rangle \approx 0.5$, showing that only half of the droplets are linked to other droplets. In contrast, with the stiff polymers, $\left\langle N_{\mathrm{d}}^{\mathrm{m}}\right\rangle=19.8$, which is similar to that for the semiflexible polymer at a 2 -fold-higher polymer concentration. Each droplet is linked to more than four other droplets. Hence, the tendency to form clusters is reduced with increasing flexibility and increases with increasing stiffness, with the reference system as the point of departure.

Figure 4 displays snapshots of some of the systems investigated. The proposed description of the structure and differences between systems IIa and IIb seem to be supported. With flexible polymers (system IIa), the droplets are reasonably homogeneously distributed, to a large degree unconnected, and partly covered by the polymers, whereas with stiff polymers (system IIb), the droplets are more heterogeneously distributed, the polymers are bridging distant droplets by spanning droplet-free regions, and nearby droplets are rarely linked to each other.

4.3. Effect of Midblock Length for Semiflexible Polymers. So far, we have only considered polymers with $N_{\mathrm{B}}=20$ middle segments. We will now examine the role of the midblock length by also considering $N_{\mathrm{B}}=10$ (system IIIa), 30 (system IIIb), and 40 (system IIIc), again with intermediate flexibility and at a polymer-droplet number ratio of $\beta=3$.

The inset of Figure 5a displays the end-to-end separation probability distribution $P\left(R_{\mathrm{ee}}\right)$ of the polymers with $N_{\mathrm{B}}=10,20$, 30 , and 40 middle segments for droplet-free polymer solutions. As expected, the maxima of these distributions are shifted to longer separations with increasing midblock length. Furthermore, the rms end-to-end separations are $\left\langle R_{\mathrm{ee}}{ }^{2}\right\rangle^{1 / 2}=104.3,155.0,192.6$, and $224.5 \AA$, respectively. Although the distributions are nearGaussian for the polymers with $N_{\mathrm{B}} \geq 20$, it is skewed for $N_{\mathrm{B}}=$ 10 , showing that the shortest block is not long enough for Gaussian statistics. In the presence of droplets, Figure 5a shows that the probability distribution $P\left(R_{\mathrm{ee}}\right)$ again is altered significantly; here, the four systems display bimodal distributions, with the two peaks having similar areas. Furthermore, the fraction of loop and 


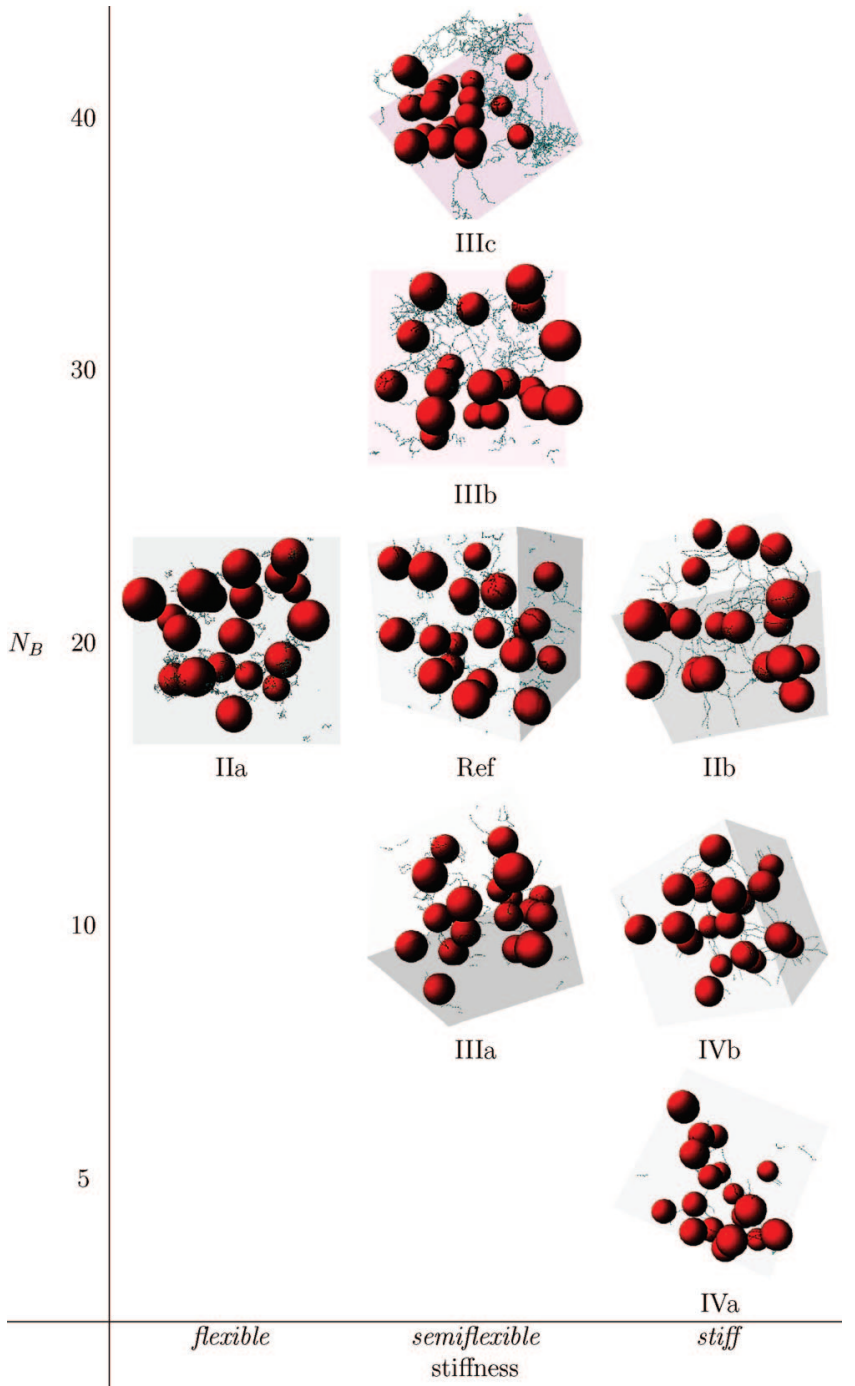

Figure 4. Snapshots of indicated systems with increasing chain stiffness along the abscissa and increasing number of segments of the middle B-block along the ordinate.

bridge conformations given in Table 2 are approximately equal. A closer inspection of Figure 5a shows that the locations of the peaks at the smaller $R_{\mathrm{ee}}$ among the four systems are relatively independent of $N_{\mathrm{B}}$, whereas the locations of the other peaks become 125, 200, 230, and $300 \AA$ for $N_{\mathrm{B}}=10,20,30$, and 40, respectively, hence increasing with $N_{\mathrm{B}}$. Thus, our data again convincingly support the identification that the peak at the shorter separation represents loop conformations and the other peak represents bridge conformations.

Figure 5a shows the droplet-droplet radial distribution functions for systems with polymers of different midblock length. For $N_{\mathrm{B}}=10$, a maximum appears at $r \approx 240 \AA$, which is followed by a minimum at $r \approx 280 \AA$; both features are induced by the added polymer. For $N_{\mathrm{B}}=20$, these features are shifted to longer distances. As mentioned earlier, there is a free-energy cost of compressing bridging polymers, leading to an effective shortrange repulsion between droplets linked by a polymer bridge, this repulsion becoming more long-ranged with increasing polymer length. This trend is, perhaps unexpectedly, interrupted when the midblock length is increased from 20 to 30 and 40 segments. Now, the amplitude of $g(r)$ at short separations increases with increasing $N_{\mathrm{B}}$. With $N_{\mathrm{B}}=40$, a very prominent peak appears at $r=185 \AA$. Hence, for the longer polymers, the droplet-droplet

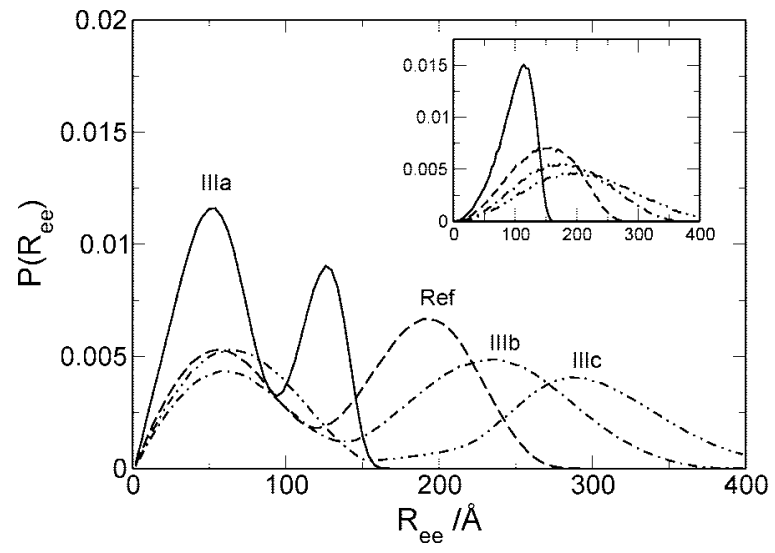

(a)

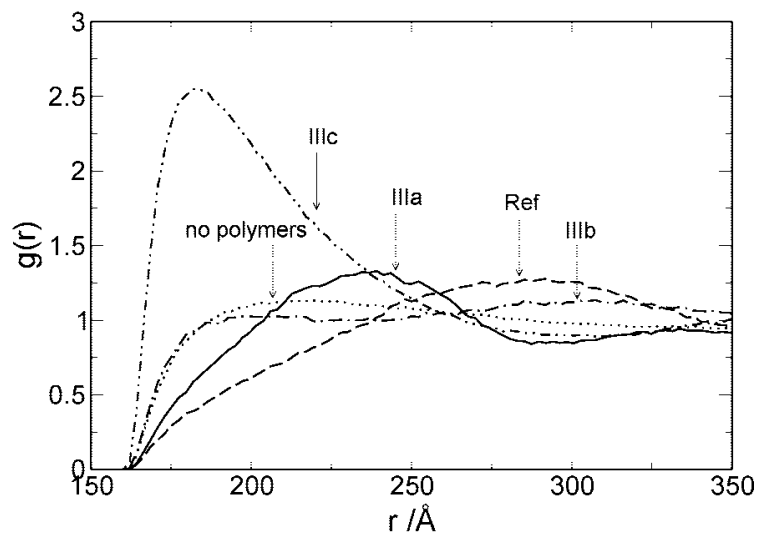

(b)

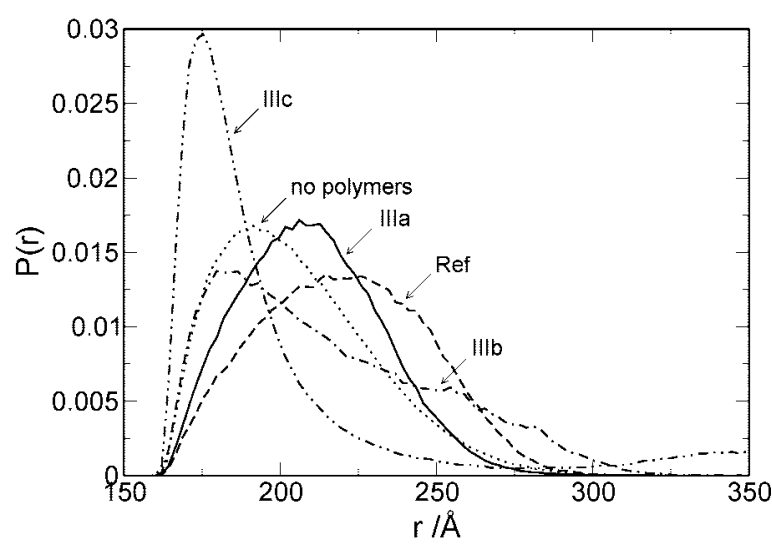

(c)

Figure 5. (a) Polymer end-to-end separation probability distribution $P\left(R_{\mathrm{ee}}\right)$, (b) droplet-droplet radial distribution function $g(r)$, and (c) droplet-droplet nearest-neighbor separation probability distribution $P(r)$ for the indicated systems containing polymers with different midblock lengths. In panel a, the inset shows the corresponding distributions for droplet-free polymer solutions.

radial distribution function suggests an extended droplet association but no strong long-range ordering.

The influence of the polymer midblock length on the nearestneighbor probability distribution is shown in Figure 5c. With $N_{\mathrm{B}}$ $=10$, the distribution is slightly displaced, and with $N_{\mathrm{B}}=20$, it is displaced even further, to larger distances, as compared to the polymer-free solution. As argued previously, this is likely due to a polymer-mediated droplet-droplet repulsion through polymers in loop conformations. With $N_{\mathrm{B}}=40$, a bimodal distribution is visible with a maximum close to contact separation 
and a weak second maximum at $r \approx 350 \AA$, supporting the notion of a droplet-rich domain.

The connectivity indicators $\left\langle N_{\mathrm{d}}^{\mathrm{m}}\right\rangle$ and $\left\langle N_{\mathrm{d}}^{\mathrm{m}}\right\rangle$ given in Table 2 display an increased cluster size as $N_{\mathrm{B}}$ is increased from 10 to 20 , a reduced cluster size as $N_{\mathrm{B}}$ is further increased to 30 , and a continued reduced cluster size as $N_{\mathrm{B}}$ is further increased from 30 to 40 . Similarly, $\left\langle N_{\mathrm{d}}^{\text {link }}\right\rangle$ shows that the largest number of links for a given droplet is obtained at $N_{\mathrm{B}}=20$ and 30 and a smaller number of links at either shorter or longer polymers. The bond order is $\left\langle N_{\mathrm{p}}^{\text {link }}\right\rangle=2.0$ with $N_{\mathrm{B}}=40$, but is considerably smaller for the other polymer midblock lengths.

Hence, as with increasing stiffness, also with increasing chain length, nontrivial structural changes occur in a solution of droplets with end-capped polymers in 3-fold number excess. Initially, at increasing chain length, the cluster size is increasing and, essentially, a single network with homogeneously distributed droplets is obtained for $N_{\mathrm{B}}=20$. For longer chains and, in particular, with $N_{\mathrm{B}}=40$, the investigated solution displayed a strong droplet-polymer segregation, which is much more prominent than that obtained with rigid polymers and $N_{\mathrm{B}}=20$. We argue that the conventional depletion attraction among the droplets arising from the presence of the polymers drives this segregation. However, we expect this segregation to be only mesoscopic; a macroscopic segregation (phase separation) would require that A-ends become dissolved in water, which, in the present model, involves an energy cost of $\sim 25 k_{\mathrm{B}} T$ per polymer. Table 2 confirms that the fraction of tail conformations remains small and that of nonadsorbed polymers is negligible, even with $N_{\mathrm{B}}=40$.

Figure 4 also displays snapshots of the sequence of the systems with increasing midblock length. The fairly weak clustering occurring with the shortest midblock (system IIIa) is observed, as well as the strong segregation with the longest midblock (system IIIc). In the latter, droplets clearly are forming essentially one big cluster and the midblocks are occupying the droplet-free volume that surrounds the cluster.

4.4. Effect of Midblock Length for Stiff Polymers. The influence of the midblock length of stiff polymers was also investigated. In the following, we will consider the midblock lengths $N_{\mathrm{B}}=5$ (system IVa), 10 (system IVb), and 20 (system $\mathrm{IIb}$ ), for stiff polymers and at a polymer-droplet number ratio of $\beta=3$.

As for $N_{\mathrm{B}}=20$, shown in Figure 3a, the end-to-end separation probability distribution $P\left(R_{\mathrm{ee}}\right)$ of the polymers with $N_{\mathrm{B}}=5$ and 10 remains the same in droplet-free solutions as in dropletcontaining solutions (data not shown). Their rms end-to-end separations are $\left\langle R_{\mathrm{ee}}{ }^{2}\right\rangle^{1 / 2}=84.8$ and $146.8 \AA$, respectively.

The droplet-droplet radial distribution functions displayed in Figure 6 a demonstrate that the system with the shortest polymers possesses a strong organization of the droplets, with a density maximum of $r \approx 215 \AA$. The strong organization remains with $N_{\mathrm{B}}=10$, now with the density maximum shifted to $r \approx 275 \AA$, whereas with $N_{\mathrm{B}}=20$, the droplets are radially less structurally organized as previously discussed.

Figure $6 \mathrm{~b}$ shows the corresponding nearest-neighbor probability distributions. With $N_{\mathrm{B}}=5$ and 10 , these distributions are skewed with a rapid decay after their maxima, which appear at somewhat smaller distances, as compared to the corresponding maxima of $g(r)$. A further increase of the chain length leads to a shift of the maximum to smaller distances, which previously has been attributed to a reorganization of the droplets, such that neighboring droplets now are not linked by bridging polymers.

Table 2 shows that essentially all polymers form bridges and all droplets constitute a single network. The average number of

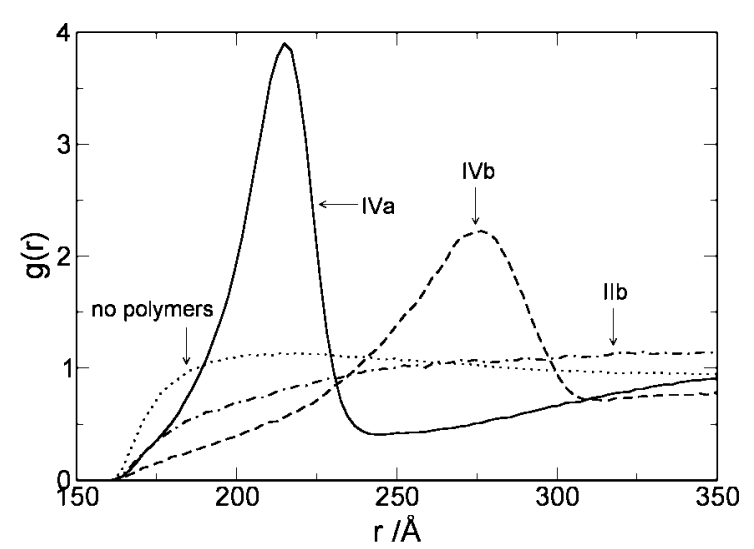

(a)

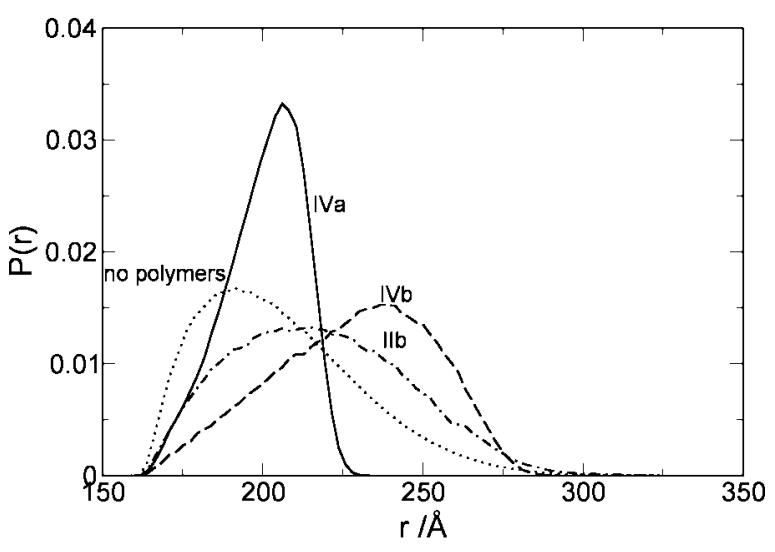

(b)

Figure 6. (a) Droplet-droplet radial distribution function $g(r)$ and (b) droplet-droplet nearest-neighbor separation probability distribution $P(r)$ for systems with stiff polymers with different midblock lengths.

droplets linked to a droplet increases from 2.5 with $N_{\mathrm{B}}=5$ to 4.6 with $N_{\mathrm{B}}=20$. With the shortest chain, the bond order is 2.35 , which demonstrates a high degree of multiple bridging. Thus, with the shortest chain, we envision a structure where each droplet is surrounded with relatively few but multiple-linked nearest neighbors. This enables a three-dimensional (3D) network that involves all droplets and all the short polymers.

The snapshot of the system with $N_{\mathrm{B}}=5$ (system IVa) shown in Figure 4a confirms the inhomogeneous distribution of the droplets and the high frequency of multiple bridging. With an increasing polymer length, the droplets become more homogeneously distributed (system IVb), and with $N_{\mathrm{B}}=20$, the polymers are so long that many neighboring droplets become unlinked, as previously discussed.

\section{Discussion}

In the previous section, we have presented simulation results of systems that contain microemulsion droplets and end-capped polymers in solution, with a focus on the formation of transient networks. We will now proceed with a discussion of some of these results.

The spatial distributions of the droplets have been described with radial distribution functions. The location, at which $g(r)$ displays its first maximum, will here be of special interest, and this location will be labeled $r_{\max }$. In the absence of polymers, $g(r)$ displayed a weak maximum at $r=r_{\max } \approx 210$ $\AA$, corresponding to a surface-to-surface separation of $50 \AA$, 


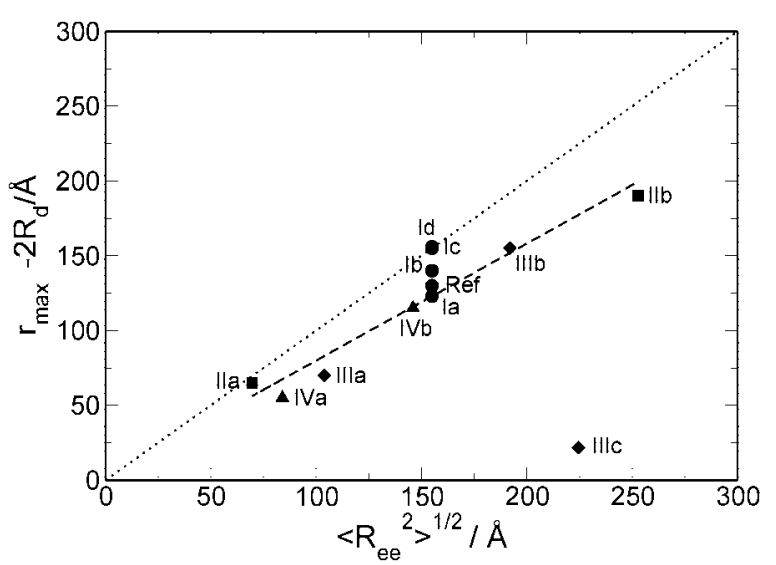

Figure 7. Droplet - droplet surface-to-surface separation at the maximum of the droplet - droplet radial distribution function $r_{\max }-2 R_{\mathrm{d}}$, as a function of the unperturbed polymer end-to-end separation $\left\langle R_{\mathrm{ee}}{ }^{2}\right\rangle^{1 / 2}$. A linear regression using all systems except system IIIc (dashed line), and the unitary slope (dotted line), are also given.

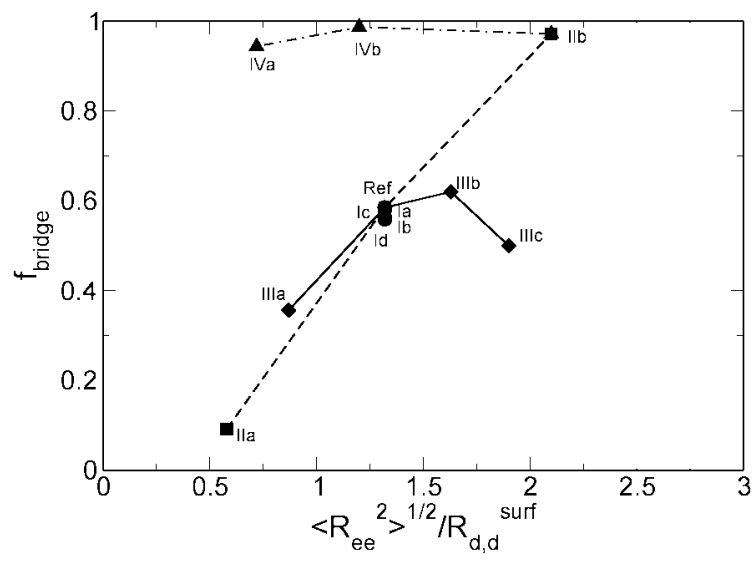

Figure 8. Average fraction of chains forming bridges as a function of the length ratio $\left\langle R_{\mathrm{ee}}{ }^{2}\right\rangle^{1 / 2} / R_{\mathrm{d}, \mathrm{d}}^{\text {surf }}$ for the indicated systems.

and then smoothly approaches unity, which is typical for a dilute solution of soft spheres possessing only excluded-volume interactions. Figure 7 displays the surface-to-surface separation at $r=r_{\max }$ in the presence of polymers as a function of the unperturbed rms end-to-end separation $\left\langle R_{\mathrm{ee}}{ }^{2}\right\rangle^{1 / 2}$. For most systems, we find a linear correlation between the surface-to-surface separation at $r=r_{\max }$ and the polymer extension, showing that the separation between neighboring droplets is strongly related to the length of the unperturbed polymer, independent of the polymer length and polymer stiffness. System IIIc constitutes an exception; here, $r_{\max }-2 R_{\mathrm{d}} \approx 20 \AA \ll\left\langle R_{\mathrm{ee}}^{2}\right\rangle^{1 / 2}=224 \AA$, which is consistent with a mesoscopic segregation where neighboring droplets are not linked. One contribution to the observation $\left\langle R_{\mathrm{ee}}{ }^{2}\right\rangle^{1 / 2}<\left(r_{\max }-2 R_{\mathrm{d}}\right)$ is the balance between (i) the free energy increase associated with the increasing structural ordering of the domains and (ii) the free energy increase of the compression of the polymers, when mixing the droplets and the polymers.

We have observed that both ends of essentially all polymers reside in droplets; however, the distribution between the loop and bridge conformations varies strongly. Figure 8 shows the variation of $f_{\text {bridge }}$ as a function of $\left\langle R_{\mathrm{ee}}^{2}\right\rangle^{1 / 2} / R_{\mathrm{d}, \mathrm{d}}^{\text {surf }}$, where the latter represents the extension of an unperturbed polymer measured by the characteristic distance between neighboring droplets when the droplets form an ordered structure spanning the solution. The values of this ratio were given in Table 1. Under most conditions, we observe an increase in the fraction of bridges from basically

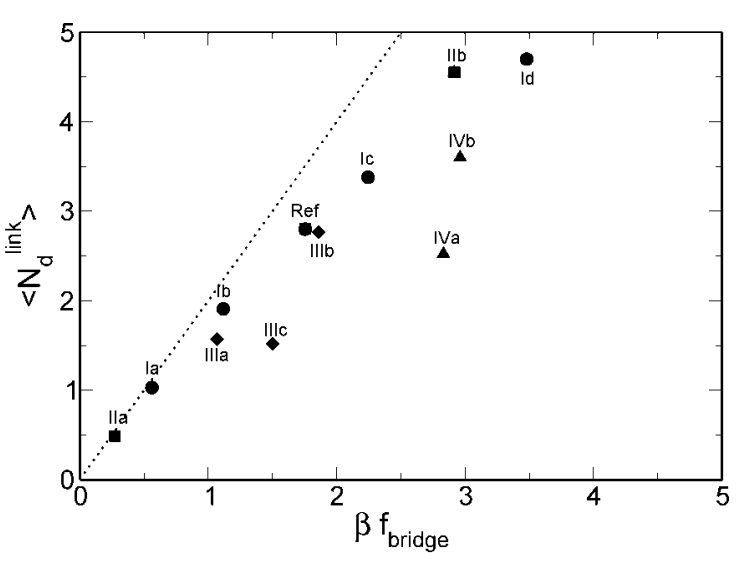

(a)

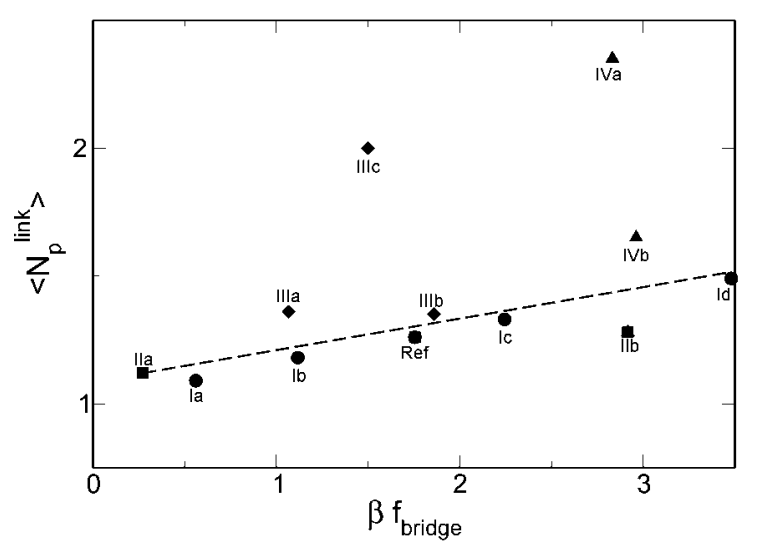

(b)

Figure 9. (a) Average number of linked droplets $\left\langle N_{\mathrm{d}}^{\text {link }}\right\rangle$ and (b) average bond order $\left\langle N_{\mathrm{p}}^{\text {link }}\right\rangle$, as a function of the ratio of the number of bridging polymers and droplets $\beta f_{\text {bridge }}$ for indicated systems. In panel a, the slope of 2 (represented by the dotted line) also is shown. In panel $b$, a linear regression using all systems except systems IIIc and IVa (represented by the dashed line) also is given.

zero to unity as $\left\langle R_{\mathrm{ee}}^{2}\right\rangle^{1 / 2} / R_{\mathrm{d}, \mathrm{d}}^{\text {surf }}$ is increased from 0.5 to 2 . Hence, loops are favored in systems where the polymers are short in comparison to the separation between neighboring droplets, whereas bridges dominate with longer polymers, with a transition appearing at $\left\langle R_{\mathrm{ee}}{ }^{2}\right\rangle^{1 / 2} / R_{\mathrm{d}, \mathrm{d}}^{\text {surf }} \approx 1$. There are two exceptions for this behavior: (i) the stiff chains in systems IVa and IVb possess basically only bridge conformations and (ii) for the mesoscopic segregated system IIIc, the fraction of bridges becomes smaller. In the former case, the polymer contour length is shorter than the persistence length making it energetically costly for a polymer to be folded such that both ends can be anchored to the same droplet. In the latter case, the droplet-polymer demixing favors loop conformations by pure geometrical arguments.

Figure 9a displays the local connectivity indicator $\left\langle N_{\mathrm{d}}^{\mathrm{link}}\right\rangle$, as a function of the ratio of the number of bridging polymers and droplets $\beta f_{\text {bridge. }}$ Again, under most conditions, a master behavior is observed. Here, $\left\langle N_{\mathrm{d}}^{\text {link }}\right\rangle$ initially displays the expected slope of 2 (expressed as a dotted line) and the slope decreases at larger values of $\beta f_{\text {bridge. }}$. Figure $9 \mathrm{~b}$ shows that the bond order $\left\langle N_{\mathrm{p}}^{\text {ink }}\right\rangle$ starts at about unity at small $\beta f_{\text {bridge }}$ (as expected) and increases to $\sim 1.5$ at $\beta f_{\text {bridge }}=3.5$. As above, systems with stiff chains (systems IVa and IVb) and with long flexible chains (system IIIc) do not conform with the general trends. Thus, at an excess of droplets, the fraction of multiple bridges is small and the number of droplets linked to a given droplet is proportional to the number of bridging polymers; however, when the polymers 


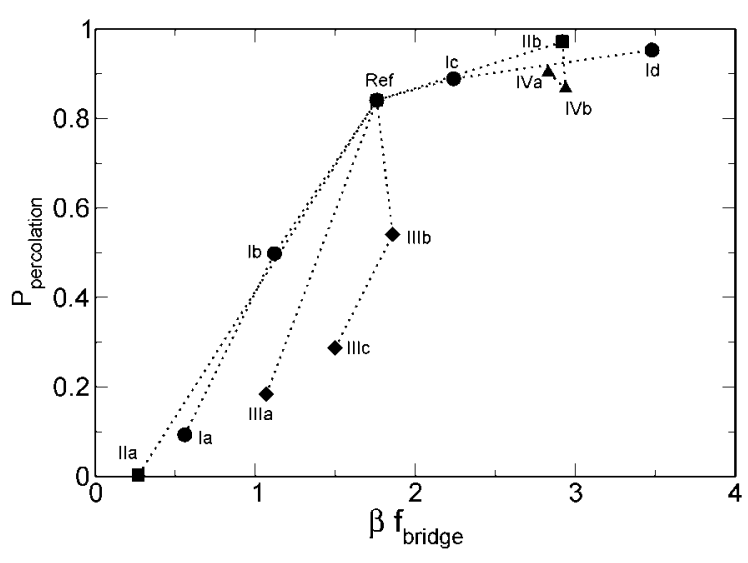

(a)

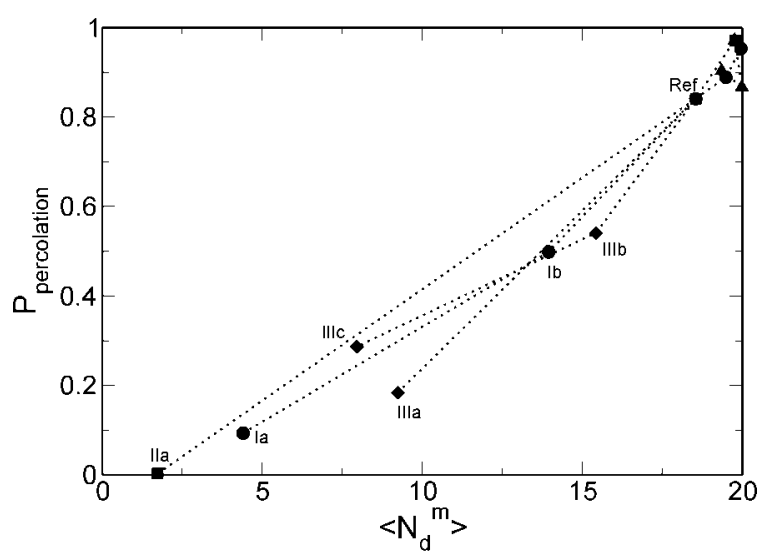

(b)

Figure 10. Percolation probability $P_{\text {percolation }}$ as a function of (a) the ratio of the number of bridging polymers and droplets $\beta f_{\text {bridge }}$ and (b) the average number of droplets forming a cluster $\left\langle N_{\mathrm{d}}^{\mathrm{m}}\right\rangle$ for indicated systems.

are in large excess, we expect that $\left\langle N_{\mathrm{d}}^{\text {link }}\right\rangle$ has leveled off and all additional bridging polymers will bridge already linked droplet pairs.

We will now continue our discussion by examining the probability that the systems are percolated. We define the percolation probability $P_{\text {percolation }}$ as the fraction of conformations for which a system is percolated in at least in one dimension. Note, under otherwise identical conditions, $P_{\text {percolation }}$ is dependent on the system size; $P_{\text {percolation }}$ is expected to decrease with increasing system size.

Figure 10a shows that the percolation probability increases rapidly as the number ratio between bridging polymers and droplets is increased from $\sim 0.5$ to $\sim 2$, whereas it approaches unity slowly when $\beta f_{\text {bridge }}>2$. System IIIc, with a strong tendency for mesoscopic segregation, and system IIIb, with a weaker tendency for mesoscopic segregation, do not follow the general trend; these systems display a reduced percolation probability. In a previous work, Surve et al. ${ }^{33}$ paid special attention to systems with $P_{\text {percolation }}=0.5$ and argued that this probability provided a reliable estimate of the percolation threshold and critical exponents. In our systems, $P_{\text {percolation }}=0.5$ is obtained between $\beta f_{\text {bridge }}=1$ and $\beta f_{\text {bridge }}=1.5$.

The relationship between $P_{\text {percolation }}$ and the overall connectivity indicator $\left\langle N_{\mathrm{d}}^{\mathrm{m}}\right\rangle$ is presented in Figure 10b. One perhaps unexpected observation is that all systems conform to the master behavior, including the systems with stiff chains and where mesoscopic phase separation occur. Obviously, percolation probability is dependent on the system variables, which are varied only through the average cluster size. The probability $P_{\text {percolation }}=0.5$ is achieved at $\left\langle N_{\mathrm{d}}^{\mathrm{m}}\right\rangle=15$, i.e., when, on average, a droplet that appears in a cluster that contains 15 droplets. The cluster size distribution function of system $\mathrm{Ib}$ given in Figure 2 is close to this percolation threshold.

The influence of the chain length on the ability of the chains to form bridges or loops has also been studied by other authors. In an experimental study, Lynch and Picule $1 l^{40}$ have reported that the increase of the midblock length enhanced the fraction of bridges formed, in accordance with our results. A decrease of the viscosity was also observed for systems where the concentration of droplets was increased, while keeping the droplet radius constant (thus increasing the number of nodes). This can be explained, given our results, as a sum of two opposing factors. Most importantly, here, an increase of the number of nodes corresponds to a decrease in the polymer-droplet number ratio, for which we have found a smaller droplet connectivity. This seems to be the main aspect for understanding the trend. Furthermore, the increase of number of nodes leads to an increase of $\left\langle R_{\mathrm{ee}}{ }^{2}\right\rangle^{1 / 2} / R_{\mathrm{d}, \mathrm{d}}^{\text {surf }}$, which we found was associated with an increase of the connectivity.

The influence of the polymers on the droplet distribution may explain some results presented previously by Antunes et al. ${ }^{39}$ It was observed that the viscosity of the systems achieved a maximum and then decreased when increasing the amount of droplets at a fixed polymer-droplet number ratio. The decrease was also more significant for longer chains than for shorter ones. Given our results, this can be viewed as a considerable decrease in the volume that is accessible to accommodate the hydrophilic blocks of the polymers, which will force the droplets to partially segregate, in this manner, decreasing the network connectivity.

\section{Conclusion}

On the basis of Monte Carlo simulations, structural properties of solutions that contain microemulsion droplets and ABA triblock copolymers have been investigated. The end-blocks were assigned properties that represented an alkyl chain with 10 carbon atoms. Our focus has been on the formation of transient networks with the droplets constituting the nodes and the polymers forming the bridges between the nodes. The network formation has been examined on a global level through cluster size and percolation analyses, and on a local level through the number of droplets linked to a given droplet and by assessing the bond order.

The fraction of polymers forming bridges was determined to have a strong dependence on the length ratio of the polymer root-mean-square (rms) end-to-end separation and the surfaceto-surface separation between neighboring droplets for a lattice arrangement of the droplets. This fraction was independent of the number of polymers and independent of the polymer length and stiffness, except for very stiff or very long polymers. With a length ratio about unity, approximately half of the polymers formed bridges. The observed distance between neighboring droplets was ca. $80 \%$ of the rms end-to-end separation of unperturbed polymers, demonstrating the organizing effect of the polymers on the droplet structure. Long flexible polymers lead to a depletion attraction among the droplets inducing a mesoscopic segregation with a decreased connectivity, whereas with polymers with a persistence length larger than the contour 
length resulted only in bridging conformations. Again, except for very stiff or very long polymers, the local connectivity indicators displayed a uniform dependence on the number bridging polymers per droplet. The percolation probability could be represented as a function of the average droplet cluster size only across all systems investigated.
Acknowledgment. Financial support by Fundação para a Ciência Tecnologia (FCT), Portugal, through Grant No. SFRH/ BD/17440/2004, and the Swedish Research Council (VR), through the Linnaeus Center of Excellence on Organizing Molecular Matter (OMM), is gratefully acknowledged.

LA801658K 